\title{
A Novel Approach to Multi-Trauma Patients with Bleeding Pelvic Fractur
}

\author{
Ethem Unal*, Omer Faruk Ozkan, Sema Yuksekdag and Ahmet Topcu \\ Health Sciences University, Umraniye Training and Research Hospital, Department of General Surgery, Istanbul, Turkey
}

Received: 㘹 August 20, 2018; Published: 制 August 27, 2018

*Corresponding author: Ethem Unal, Health Sciences University, Umraniye Training and Research Hospital, Department of General Surgery, Istanbul, Turkey

\section{Summary}

With concomitant pelvic fracture, differentiating between pelvic-bleeding and intra-abdominal hemorrhage is critical to the initial decision-making and management of the patient. In the present compact review, we interrogated the current successful methods in dealing with bleeding pelvic fractures.

Keywords: Pelvic Fracture; Trauma; Bleeding; Packing

\section{Introduction}

Bleeding from pelvic fracture, especially in patients with multiple trauma injuries, is a difficult problem facing the general practitioner working at Emergency Room (ER). Orthopedist, general surgeon, neurosurgeon, urologist and interventional radiologist are the main consultants taking care of these kind of patients. Recently, there have been many changes in practice to predict and limit hemorrhage in the patient with a pelvic fracture, and multidisciplinary approach has improved the outcome and overall survival [1]. Institutional guidelines or trauma protocols should be well-established especially in advanced trauma centers.

The first thing to do in these patients is to understand if the case is hemodynamically stable or unstable [2]. Multiple intravenous (iv) accesses should be ready in use and Foley catheter should be inserted in the monitorized patient. In hemodynamically stable patients, simple orthopedic fractures and any possible accompanying intracranial pathology can be excluded by using computerized tomography (CT) scans from cranium to the pelvis and X-rays of all suspected extremities. Then, the ER team can emphasize particularly on the pelvic fracture. The ongoing bleeding should be further interrogated. Furthermore, the accompanying intraabdominal and/or retroperitoneal pathology should also be evaluated in detail. FAST (focused assessment with sonography in trauma) can be a useful tool in the evaluation of precordial and abdominal cavities [3]. However, its dependence on the personal experience seems to be a major disadvantage in comparison with more detailed CT interpretations. Hemodynamically unstable patients with suspected intraabdominal bleeding should undergo emergent exploration.

Pelvic stabilization has been attempted for years to control bleeding by decreasing the pelvic volume and supplying tamponade effect [4]. Initially used pneumatic antishock garments have largely fallen out of favor as they lead to compartment syndrome, and they are bulky, difficult to apply and interfere with physical examination. In case of unstable pelvic injuries, the initial application of external pelvic fixation and pelvic C-clamp can be tried in an attempt to control hemorrhage associated with pelvic fracture [5]. However, an emergent pelvic angiography and embolization should be planned after nonpelvic sources of blood loss have been ruled out. A CT finding of arterial iv contrast extravasation in the pelvis is another indication for pelvic angiography and embolization regardless of the hemodynamic status. However, it only controls arterial bleeding and indicated in only up to $10 \%$ of cases with the pelvic instability.

The exclusion of intraabdominal bleeding is vital in patients with pelvic fracture. FAST and abdominal CT are the best tools here, and replaced the historically used diagnostic peritoneal tap or lavage which are known with their high false negative or positive rates [6]. As the indications for explorative laparotomy in cases with pelvic fracture are the same for all trauma patients, the concomitant bleeding from pelvis and abdomen should be differentiated, and each should be treated. If abdominal exploration reveals a specific organ injury, repair can be tried in case of stabilization of the vitals 
intraoperatively. However, intraabdominal or retroperitoneal packing is currently accepted as the golden standand approach in unstable multiple trauma patients with ongoing abdominal bleeding where the major vessels are severely injured [7]. This procedure saves time till the patient gains hemodynamic stability and the physician decide what to do the next. Therefore, the procedure is also called as 'damage control surgery' [7,8]. In case of presacral bleeding accompanying multiple pelvic fractures, packing of the pelvic cavity can be life-saving. Pelvic packing can also be used in unstable patients without intraabdominal pathologies [9]. In these cases, exploration starts with a midline incision from umbilicus toward the pelvis, and bladder is retracted away from the fracture and three to five laparotomy pads are soaked into the retroperitoneal space on each side (toward the iliac vessels). Packing in combination with external mechanical fixation of the pelvis is reported to be successful as well. The second-look surgery can be planned in first to third postoperative days, and the laparotomy pads are removed carefully.

\section{References}

1. Tesoriero RB, Bruns BR, Narayan M, Dubose J, Guliani SS, et al. (2017) Angiographic embolization for hemorrhage following pelvic fracture: Is it time for a paradigm shift? J Trauma Acute Care Surg 82(1): 18-26.
2. Guillamondegui OD, Pryor JP, Gracias VH, Gupta R, Reilly PM, et al. (2002) Pelvic radiography in blunt trauma resuscitation: a diminishing role. J Trauma 53(6): 1043-1047.

3. Carter JW, Falco MH, Chopko MS, Flynn WJ Jr, Wiles Iii CE, et al. (2015) Do we really rely on fast for decision-making in the management of blunt abdominal trauma? Injury 46(5): 817-821.

4. Marzi I, Lustenberger $T$ (2014) Management of Bleeding Pelvic Fractures. Scand J Surg 103(2): 104-111.

5. Heini PF, Witt J, Ganz R (1996) The pelvic C-clamp for the emergency treatment of unstable pelvic ring injuries. A report on clinical experience of 30 cases. Injury 27(1): 38-45.

6. Stahel PF, Burlew CC, Moore EE (2017) Current trends in the management of hemodynamically unstable pelvic ring injuries. Curr Opin Crit Care 23(6): 511-519.

7. Burlew CC (2017) Preperitoneal pelvic packing for exsanguinating pelvic fractures. Int Orthop 41(9): 1825-1829.

8. Cho J, Benjamin E, Inaba K, Lam L, Demetriades D (2018) Severe Bleeding in Pelvic Fractures: Considerations in Planning Damage Control. Am Surg 84(2): 267-272.

9. Li Q Dong J, Yang Y, Wang G, Wang Y, et al. (2016) Retroperitoneal packing or angioembolization for haemorrhage control of pelvic fractures--Quasi-randomized clinical trial of 56 haemodynamically unstable patients with Injury Severity Score $\geq 33$. Injury 47(2): 395-401.
To Submit Your Article Click Here:

Submit Article

DOI: $10.32474 /$ OSMOAJ.2018.01.000123

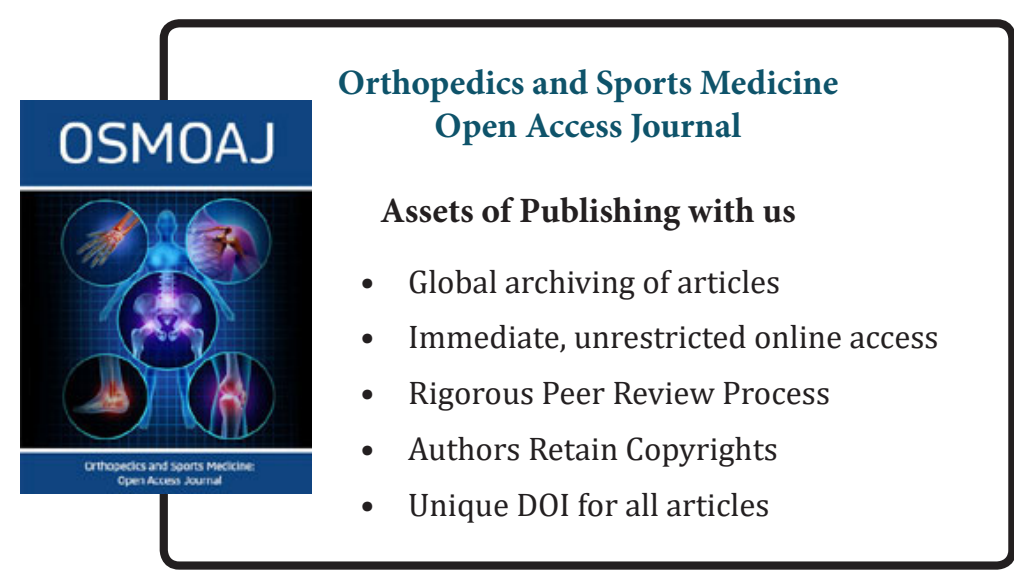

